\title{
Editorial
}

\section{Curriculum development and the future of anesthesia training programs}

\author{
David Parsons MD FRCPC
}

I $\mathrm{N}$ this issue of the Journal, Dr. Anne Wong ${ }^{1}$ presents a detailed description of the process of curriculum development. The importance of such development should not be underestimated as our profession and specialty undergo challenges and change.

Historically, anesthesia educators in many of our Canadian University Centres (including my own) have designed curriculum for our residency programs in isolation from most of our colleagues, and from many of the other programs in the country. We have made academic plans with a keen eye on the latest Royal College written and oral examination content and have often heard following presentations at academic days or grand rounds the comment that the topic, while interesting, will not be on the examination - and hence not part of the curriculum. While having an examination-driven curriculum has been practical for our trainees, this is not necessarily beneficial for the future of our profession. If we were to develop curriculum that was relevant to contemporary practice and standardize it throughout the 17 (and growing) training centres, we could then prepare a blueprint for our examinations that had true validity with our trainees and our faculty.

The release of the revised CanMEDS $2005^{2}$ roles presents just such an opportunity. As anesthesiology works to customize these roles for the "Specialty Training Requirements" and "Objectives of Training," we may well begin the process of curriculum driving the examinations. Our efforts with respect to some roles have been successful, such as Medical Expert and Scholar, but in areas such as Health Advocate and Manager, we as well as the other specialties have more work to do. The refined language and greater practicality of the second iteration of the CanMEDS concept should make this job easier than in the past. Department heads as well as the faculty as a whole need to support our program directors and their volunteers in curriculum development.

As important as our specialty training is to the future, we must also look closely at the curriculum that we design for the undergraduate exposure to anesthesiology. One of our responsibilities as educators to our profession is to recruit the best and brightest medical students to our specialty, but also we have the mandate to inculcate practical physiology and pharmacology into all graduating physicians. The loss of undergraduate mandatory anesthesia rotations in some medical schools needs urgent and ongoing attention.

And how to make our undergraduate rotations untouchable? We have the work of the University of Toronto group and their use of the high fidelity simulator in undergraduate education to which we may refer. ${ }^{3}$ If we agree to develop a national undergraduate anesthesia program with emphasis on core competencies in such areas as airway, breathing and circulation, as well as basic competencies in resuscitation, we will create a need that all medical schools and students will see as mandatory. As simulation programs gain stability (personnel and financial) from the use of mandatory residency rotations, diversification of participants and as possible evaluation tools, ${ }^{4}$ we can use the very popular face validity of simulation backed up with science, to truly design multi-facetted rotations that deliver essential content to our medical schools.

For our residency programs we also need to continually re-evaluate the content and presentation of our curriculum. Not only does simulation challenge

From the Department of Anesthesiology, Vancouver Hospital and Health Sciences Centre, Vancouver, British Columbia, Canada. Address correspondence to: Dr. David Parsons, Department of Anesthesiology, Vancouver Hospital and Health Sciences Centre, JP2 Room 2449, 910 West 10 ${ }^{\text {th }}$ Avenue, Vancouver, British Columbia V5Z 4E3, Canada. Phone: 604-875-4304; Fax: 604-875-5209; E-mail: david.parsons@vch.ca 
our standard paradigm of operating room experience being superior to all other kinds of learning in developing competencies, it also signals the effect that evolving technology is having on anesthesiology. We need to look critically, as proposed by Dr. Wong, at the content of our residencies with respect to inclusion of transesophageal echocardiography, the use of imaging techniques for nerve blockade and vascular access, crisis resource management, and of course the CanMEDS roles that up to now have been less than adequately emphasized.

Changes to undergraduate and graduate curricula also need to be managed by specially trained anesthesiologists. Academic centres have been fortunate to this point in having a number of faculty focus on educational theory through master's programs, both locally and internationally. Our departments need to encourage this trend and appoint directors of education with advanced degree standing so that new approaches to teaching and learning, as well as more effective use of traditional methods, are promoted within our faculty. ${ }^{5}$

Well-educated faculty directing improved curriculum in our undergraduate and residency programs will be an important third leg of the clinical care, research and education stool representing the specialty of anesthesiology.

\section{L'élaboration d'un cur- sus et l'avenir des pro- grammes de formation en anesthésie}

Dans le présent numéro du Journal, la Dr Anne Wong ${ }^{1}$ présente une description détaillée de l'élaboration d'un cursus. L'importance de cette entreprise ne doit pas être sous-estimée, car notre profession et notre spécialité sont soumises à des défis et des changements.

Historiquement, les professeurs en anesthésie de nombreux centres universitaires canadiens, y compris le mien, ont élaboré des cursus pour les programmes de résidence isolément de leurs collègues et des nombreux programmes d'autres pays. Nous avons fait des plans pédagogiques en nous intéressant au contenu du dernier examen oral et écrit du Collège royal et nous avons souvent entendu, après des présentations lors de journées de formation ou de séances scientifiques, le commentaire voulant qu'un sujet, même intéressant, ne soit pas abordé à l'examen - et, par conséquent, ne fasse pas partie du cursus. Le cursus axé sur l'examen a été pratique pour les résidents, mais il n'est pas nécessairement avantageux pour l'avenir de notre profession. Si nous nous préparons à élaborer un cursus utile à la pratique contemporaine et à le standardiser dans les 17 centres existants et à venir, nous devons alors préparer des prototypes de nos examens réellement valides pour nos résidents et nos professeurs.

La publication d'une révision des rôles des CanMEDS $2005^{2}$ présente justement cette possibilité. Comme l'anesthésiologie cherche à adapter ces rôles pour les «Besoins en formation spécialisée» et les «Objectifs de formation», nous pouvons tout aussi bien instaurer des examens axés sur le cursus. Nos efforts en ce qui concerne certains rôles ont été couronnés de succès, comme ceux d'expert et de chercheur médical, mais dans des domaines comme ceux de conseillers ou de gestionnaires de la santé, nous avons plus de travail à faire, à l'instar d'autres spécialités. Le langage plus précis et le caractère plus pratique de la deuxième édition du concept des CanMEDS devraient faciliter le travail cette fois. Les directeurs de départements et les facultés universitaires dans leur ensemble doivent appuyer nos directeurs de programmes et leurs collaborateurs dans l'élaboration du cursus.

Aussi importante que soit la formation à notre spécialité pour le futur, nous devons aussi examiner attentivement le cursus planifié pour l'exposition des étudiants de médecine à l'anesthésie. Une de nos responsabilités comme éducateurs est de recruter les étudiants les meilleurs et les plus aptes, mais aussi d'enseigner les principes pratiques de la physiologie et de la pharmacologie à tous les étudiants de médecine. La perte des rotations obligatoires en anesthésie dans certaines écoles de médecine nécessite une attention urgente et permanente.

Comment maintenir les rotations en anesthésie pour les étudiants de médecine ? Nous pouvons nous référer au travail de l'université de Toronto et au simulateur de grande fidélité utilisé dans la formation des médecins. ${ }^{3}$ Si nous acceptons d'élaborer un programme national d'anesthésie de premier cycle en insistant sur les compétences fondamentales en intubation, respiration et circulation, autant que sur les aptitudes de base en réanimation, nous créerons un besoin que toutes les écoles de médecine et leurs étudiants considéreront comme essentiel. À mesure que les programmes de simulation gagnent en stabilité (personnel et finance) avec les rotations obligatoires pendant 
la résidence, la diversification des participants et les outils d'évaluation possibles, ${ }^{4}$ nous pouvons utiliser la très populaire simulation de validité apparente confirmée par la science pour concevoir fidèlement des rotations à multiples facettes qui peuvent délivrer le contenu essentiel de nos écoles de médecine.

Quant aux programmes de résidence, nous devons aussi en réévaluer constamment le contenu et la présentation. La simulation ne vient pas seulement bousculer notre expérience habituelle de la salle d'opération vue comme étant supérieure à toute autre forme d'apprentissage pour développer des compétences, elle montre aussi l'effet de la technologie en évolution sur l'anesthésiologie. Il faut avoir un regard critique, comme l'a proposé la Dr Wong, sur le contenu des résidences quant à l'inclusion de l'échocardiographie transœsophagienne, l'usage des techniques d'imagerie pour le blocage nerveux et l'accès vasculaire, la gestion des ressources de crise et, bien sûr, les rôles des CanMEDS qui ont été, jusqu'à maintenant, très peu soulignés.

Les changements à apporter au cursus médical et post-médical doivent relever aussi d'anesthésiologistes spécialement formés. À cet égard, les centres universitaires ont été privilégiés d'avoir de nombreux professeurs dont la formation était axée sur la théorie pédagogique grâce à des programmes de maîtrise suivis au pays et à l'étranger. Nos départements doivent encourager la nomination de directeurs de l'enseignement de formation supérieure pour que les nouvelles approches d'enseignement et d'apprentissage, et l'usage plus efficace des méthodes traditionnelles soient promus dans nos universités. ${ }^{5}$

La présence de directeurs universitaires bien formés régissant un cursus amélioré pour les programmes de médecine et de résidence constituera un important pilier pour soutenir les soins cliniques, la recherche et l'enseignement qui représentent la spécialité de l'anesthésiologie.

\section{References}

1 Wong $A$. Curriculum development in anesthesia: basic theoretical principles. Can J Anesth 2006; 53: 950-60.

2 Royal College of Physicians and Surgeons of Canada. The CanMEDS roles framework. Ottawa, Canada. CanMEDS, 2005. Cited May 11, 2006. Available from URL; http://rcpsc.medical.org/canmeds/index.php.

3 Morgan PJ, Cleave-Hogg D, McIlroy J, Devitt JH. Simulation technology: a comparison of experiential and visual learning for undergraduate medical students. Anesthesiology 2002; 96: 10-6.

4 Savoldelli GL, Naik VN, Joo HS, et al. Evaluation of patient simulator performance as an adjunct to the oral examination for senior anesthesia residents. Anesthesiology 2006; 104: 475-81.

5 Centre for Medical Education. University of Dundee. Dundee Scotland. Cited May 12, 2006. Available from URL; http://dundee.ac.uk/meded/frames/home. html. 